\title{
Gauss Maps and Symmetric Spaces
}

\author{
J.-H. Eschenburg*
}

7th September 2007

\section{Introduction}

One of the classical tools for the theory of $m$-dimensional submanifolds $M$ in euclidean $n$-space $\mathrm{E}=\mathbb{R}^{n}$ is the Gauss map $N$. It assigns to each point $x \in$ $M \subset$ E its normal space $N_{x}=\left(T_{x}\right)^{\perp}$ and takes values in the Grassmannian $\mathrm{Gr}=\mathrm{Gr}_{k}\left(\mathbb{R}^{n}\right)$ of all (possibly oriented) $k$-dimensional linear subspaces in $\mathbb{R}^{n}$ where $k=n-m$ is the codimension of $M$. We briefly discuss the question if the submanifold can be recovered from its Gauss map, and we ask for classes of submanifolds where this happens. Then we restrict attention to a class of submanifolds where much more is valid: these submanifolds are recovered just from the image of their Gauss maps.

In the hypersurface case $k=1$, the range of the Gauss map is the $m$ sphere which has the same dimension as $M$. In higher codimension we always have $\operatorname{dim} M<\operatorname{dim} \mathrm{Gr}$, but sometimes the values of $N$ actually lie in an $m$ dimensional totally geodesic subspace of Gr; e.g. this happens for complex hypersurfaces of $\mathbb{C}^{p}$ where $N$ takes values in $\mathbb{C P}^{p-1} \subset \mathrm{Gr}_{2}\left(\mathbb{R}^{2 p}\right)$. Further, if $M \subset \mathrm{E}$ is an extrinsic symmetric space, i.e. it is invariant under the reflection at each of its normal spaces, then the Gauss image of $M$ is also totally geodesic. According to Nikolaewskii [15], there are no other totally geodesic Gauss images in $\mathrm{Gr}$, and the last mentioned case is completely rigid:

Theorem. Let $M_{o} \subset \mathrm{E}$ be an $m$-dimensional (full) irreducible extrinsic symmetric submanifold other than the sphere $\mathrm{S}^{m}$, and let $N_{o}: M_{o} \rightarrow \mathrm{Gr}$ be its Gauss map. Let $M \subset \mathrm{E}$ be any submanifold of the same dimension

*eschenburg@math. uni-augsburg.de 
$m$ with non-constant Gauss map $N: M \rightarrow$ Gr. Assume $N(M) \subset N_{o}\left(M_{o}\right)$. Then up to translations and scaling, $M$ is an open subset of $M_{o}$.

In the present paper we give a conceptual proof of this part of Nikolaewskii's theorem (the original proof involves extended matrix computations with many different cases). This purely local theorem is very remarkable: It determines a (small piece of a) submanifold only from its Gauss image. A similar statement for real or complex hypersurfaces would be obviously false; the Gauss image is always contained in the sphere or complex projective space which does not restrict the shape of the submanifold.

The paper is organized as follows. In sections $2-5$ we introduce the "main players": fundamental forms, Gauss maps and extrinsic symmetric spaces. The theorem is proved in Sections 6 and 7. One of the main tools for the proof is Lemma 2 which was also used in [2] and goes back to Naitoh (cf. [14]). Note that all our considerations are local, therefore we do not have to distinguish between embeddings and immersions.

It is a pleasure to thank V. Matveev for hints and discussion.

\section{Fundamental forms and Gauss map}

Differential geometry began with the study of submanifolds, curves and surfaces in euclidean 3-space, and even today these are the main objects used to explain general geometric ideas. The theory of submanifolds $M$ in euclidean $n$-space $\mathrm{E}=\mathrm{E}^{n}$ (cf. [1] for a recent approach) has two different aspects: inner and outer geometry. Inner geometry is based on the interior distance between points in $M$ where the distance is measured by arc length of curves within $M$ while outer geometry describes how tangent or normal spaces move from point to point inside the ambient space. They correspond to the two main invariants, the first and second fundamental forms $g$ and $\alpha$ :

$$
\begin{aligned}
g(v, w) & =\langle v, w\rangle, \\
\alpha^{\xi}(v, w) & =\left\langle\partial_{v} w, \xi\right\rangle=-\left\langle w, \partial_{v} \xi\right\rangle=\left\langle A_{\xi} v, w\right\rangle
\end{aligned}
$$

for any normal field $\xi$ and any two tangent vector fields $v, w$ on $M$. For the simplest objects of differential geometry, the planar curves, the two invariants are just numbers, arc length and curvature. C.F. Gauss derived a relation between the two fundamental forms which is now called Gauss equation:

$$
R=\alpha \wedge \alpha
$$


where $R$ is the Riemannian curvature tensor (a second order expression in g). It was created by Gauss' student B. Riemann who isolated the first aspect and created an inner geometry on manifolds which no longer need to be embedded. The existence and uniqueness theorem for submanifolds says that one only needs to prescribe the two fundamental forms on $M$ in order to encode the full submanifold geometry; in fact, the relations between the fundamental forms discovered by Gauss, Codazzi and Ricci are necessary and sufficient to warrant an embedding which is unique up to rigid motions.

Yet in some sense there is a more fundamental invariant for submanifolds in euclidean space, $M \subset \mathrm{E}$, expressing directly the point-dependence of the normal spaces. This is the Gauss map which has been studied before by Euler and others. It assigns to each point $x \in M$ its normal space $N_{x}:=N_{x} M=\left(T_{x} M\right)^{\perp} \subset \mathrm{E}$. For surfaces in $\mathrm{E}^{3}$ and more generally for $m$ dimensional submanifolds of $E^{m+1}$ (hypersurfaces) the normal space is just a line, i.e. an element of real projective $m$-space $\mathbb{R P}^{m}$, and if the normal lines are orientated, the Gauss map takes values in the $m$-sphere $\mathbf{S}^{m}$, the 2-fold cover of $\mathbb{R P}^{m}$. Gauss had a practical reason to study this map: Being the astronomer Royal of the kingdom of Hannover (now the German state Niedersachsen), he was responsible for the surveying of this country 1821-1825, and the Gauss map of the earth surface (the zenith direction) is important for surveying since it determines the geographical coordinates. The triangulation used by Gauss was shown on the last German 10 Mark bill.

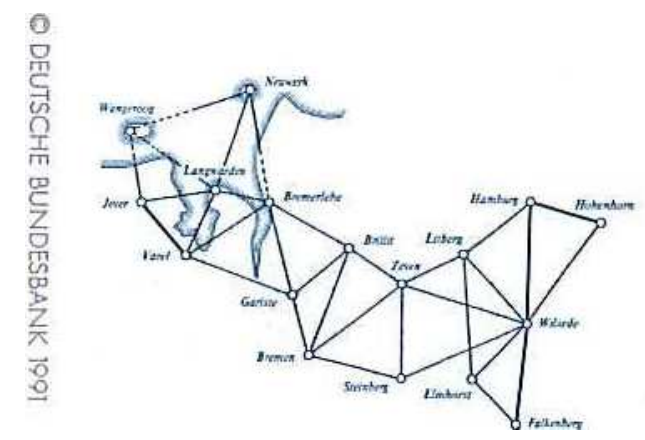

In general, the Gauss map of a submanifold $M^{m} \subset \mathrm{E}^{n}$ is a map into the Grassmannian $\mathrm{Gr}=\mathrm{Gr}_{k}\left(\mathrm{E}^{n}\right)$ of $k$-dimensional linear subspaces of $\mathrm{E}^{n}$ where $k=n-m$ is the codimension; if $M$ is oriented, one may put $\mathrm{Gr}$ the oriented Grassmannian. ${ }^{1}$ Since the derivative of this map $N: M \rightarrow \mathrm{Gr}$ measures how

\footnotetext{
${ }^{1}$ The oriented Grassmannian consists of the $k$-dimensional oriented subspaces of $\mathrm{E}$ (each
} 
the normal space is moving inside the ambient space, it should be essentially the second fundamental form. In fact, the tangent vectors of $\mathrm{Gr}$ at $N_{x} \in \mathrm{Gr}$ are the linear maps $f: N_{x} \rightarrow N_{x}^{\perp}=T_{x}{ }^{2}$, and in particular, the tangent vector $\partial_{v} N \in T_{N_{x}}$ Gr for any $v \in T_{x}$ is the linear map

$$
\partial_{v} N: N_{x} \ni \xi \mapsto\left(\partial_{v} \xi\right)^{T}=-A_{\xi} v \in T_{x} .
$$

\section{3 "Inverting" the Gauss map?}

We want to consider the following problem: To which extend does the Gauss map of a submanifold $M \subset \mathrm{E}$ determine its shape? More precisely, given the Gauss map $N: M \rightarrow$ Gr together with the induced metric on $M$ (first fundamental form), can we recover the embedding $M \hookrightarrow \mathrm{E}$ ? On the first sight, the answer seems easy: Since the second fundamental form is the derivative of $N$, both fundamental forms are given and the submanifold is determined by the existence and uniqueness theorem. But this is false! Conterexamples are obtained from minimal surfaces, i.e. surfaces in $\mathrm{E}^{3}$ with $H:=\frac{1}{2}$ trace $\alpha=0$. They allow an isometric deformation preserving the Gauss map; the best known example is the deformation of the catenoid into the helicoid. ${ }^{3}$ Hence the same metric and Gauss map on the parameter manifold may allow several non-congruent isometric embeddings. ${ }^{4}$

What was wrong with the argument? The given data consist of an abstract Riemannian manifold $M$ and a smooth map $N: M \rightarrow$ Gr. In order to obtain the second fundamental form from (2), we need to identify the abstract tangent bundle $T M$ with the subbundle $N^{\perp} \subset M \times$ E. The corresponding

subspace appears doubly, with the two possible orientations). It is a 2 -fold cover of the ordinary Grassmannian; for $k=1$ this is the covering $\mathrm{S}^{m} \rightarrow \mathbb{R} P^{m}$.

${ }^{2}$ Subspaces near $N_{x}$ are graphs of linear maps $f: N_{x} \rightarrow N_{x}^{\perp}=T_{x}$.

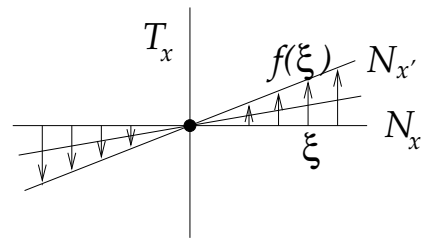

${ }^{3} \mathrm{http}$ ///en.wikipedia.org/wiki/Catenoid

${ }^{4}$ However, this cannot happen for non-minimal surfaces in $\mathrm{E}^{3}$ : The Weingarten map $A$ (being a $2 \times 2$-matrix) satisfies $0=A^{2}-(\operatorname{trace} A) A+(\operatorname{det} A) I=A^{2}-2 H A+K I$. From $g$ and $N$ we obtain $K$ and $A^{2}=d N^{t} d N$ and $H^{2}=\frac{1}{4}$ trace $\left(A^{2}\right)+\frac{1}{2} K$, and if $H \neq 0$, we recover $A=\frac{1}{2 H}\left(A^{2}+K I\right)$. Thus the embedding is uniquely determined by $g$ and $N$. 
orthogonal isomorphism $F: T M \rightarrow N^{\perp}$ needs to be parallel with respect to the Levi-Civita connection on $T M$ and the projection connection of $N^{\perp}$, and moreover, the expression $\left(\partial_{v}(F w)\right)^{\perp}=\alpha(v, w)$ must be symmetric for any two tangent vector fields $v, w$ on $M{ }^{5}$ For generic data it is impossible to construct such a map. The precise obstructions for a map $N: M \rightarrow \mathrm{Gr}$ to be the Gauss map of an embedding of $M$ seem to be unknown.

We can find positive answers for certain classes of submanifolds. One such class is formed by the surfaces in $\mathrm{E}^{3}$ with fixed nonzero constant mean curvature $H$, say $H=\frac{1}{2}$. Their Gauss map is harmonic [17], and for each $\mathrm{S}^{2}$-valued harmonic map $N$ on a simply connected two-dimensional Riemann surface $M$ there is (up to translations) exactly one immersion $M \rightarrow \mathrm{E}^{3}$ with $H=\frac{1}{2}$ and Gauss map $N$. Ironically it is the same isometric deformation which provides the counterexamples in the minimal surface case $(H=0)$ and which in the case $H=\frac{1}{2}$ is used to reconstruct the surface from its Gauss map. ${ }^{6}$ In higher dimensions and codimensions we only know one class of examples which we are going to describe next.

If $M \subset \mathrm{E}$ is a hypersurface $(k=1)$, then $M$ and $\mathrm{Gr}=\mathrm{S}^{m}$ have the same dimension $m$, a very important geometric property: recall that the Gauss-Kronnecker curvature of $M$ is the determinant of $d N$ which has many implications. This is no longer true for codimension $k \geq 2$ since $\operatorname{dim} \mathrm{Gr}=$ $\operatorname{dim} \operatorname{Hom}(N, T)=k m>m=\operatorname{dim} M$. But in some cases we are able to find a totally geodesic submanifold $Q \subset \mathrm{Gr}$ with $\operatorname{dim} Q=m$ and $N(M) \subset Q$. E.g. if $\mathrm{E}=\mathbb{R}^{2 p}=\mathbb{C}^{p}$ and $M \subset \mathbb{C}^{p}$ is a complex submanifold with dimension $m=2 p-2$ (complex hypersurface), then $N_{x}$ is always a complex line and thus $N$ takes values in complex projective space $\mathbb{C P}^{p-1}$ which is a totally geodesic $m$-dimensional submanifold of $\mathrm{Gr}_{2}\left(\mathbb{R}^{2 p}\right)$.

In fact, Nikolaewskii [15] has classified the situation where such $Q$ exists. Besides real and complex hypersurfaces there is a third class which is related to extrinsic symmetric spaces (see next section). Using extended matrix

\footnotetext{
${ }^{5}$ It is easy to see that these two requirements are also sufficient since the $\mathbb{R}^{n}$-valued 1form $F$ is closed and can be integrated. If $(M, g)$ allows two different isometric embeddings with the same Gauss map $N$, the two parallel isomorphisms $F, \tilde{F}: T M \rightarrow N^{\perp}$ yield a parallel and orthogonal automorphism $F^{-1} \circ \tilde{F}$ of $T M$. Generically, i.e. if the holonomy group is $S O(m)$ with $m>2$, such an automorphism must be trivial, thus an isometric embedding with prescribed Gauss map is unique. In the case of minimal surfaces described above, the automorphism is rotation by a constant angle. A similar phenomenon occurs in higher dimensions for pluri-minimal submanifolds, cf. [7].

${ }^{6}$ This is the famous Sym-Bobenko Formula; see [6] for details and generalization.
} 
computations, Nikolaewskii could show that this last case is completely rigid: the Gauss map image determines the submanifold uniquely. We wish to give a conceptual proof for this theorem (as stated in the introduction).

\section{Extrinsic symmetric spaces}

A symmetric space is a Riemannian manifold $M$ with an isometric point reflection (symmetry) at every point $x \in M$, i.e. there is an isometry $s_{x}$ of $M$ with $s_{x}(x)=x$ and $\left(d s_{x}\right)_{x}=-I$. We are interested in a submanifold version of this notion which is defined as follows.

For every submanifold $M \subset \mathrm{E}$ and any point $x \in M$ we let $\tau_{x}$ be the normal reflection at $x$, i.e. the affine isometry of $\mathrm{E}$ fixing $x$ whose linear part (differential) $\tau_{x *}$ has eigenvalues 1 on $N_{x}$ and -1 on $T_{x}$. A submanifold $M \subset \mathrm{E}$ is called extrinsic symmetric if it is preserved by all these normal reflections $\tau_{x}, x \in M$ (cf. [1], Section 3.7). Clearly, an extrinsic symmetric space with its induced Riemannian metric is a symmetric space where the symmetry at $x$ is $\left.\tau_{x}\right|_{M}$. Examples are euclidean spheres $\mathrm{S}^{m} \subset \mathrm{E}^{m+1}$, the orthogonal group $O(n) \subset \mathbb{R}^{n \times n}$ or the Grassmannians where a $k$-dimensional linear subspace is replaced with the orthogonal reflection at this subspace and the receiving space $\mathrm{E}$ consists of all symmetric $n \times n$-matrices. ${ }^{7}$ In fact, most symmetric spaces (so called symmetric $R$-spaces, [12]) allow such an embedding.

The hermitian symmetric spaces are of particular interest. These are symmetric spaces $M$ with a Kähler structure $J$ such that all symmetries are holomorphic. Then at any $x \in M$, the complex structure $J_{x}$ on $T_{x} M$ is a skew symmetric derivation of the curvature tensor at $x$ and hence an element of the isotropy Lie algebra $\mathfrak{g}_{x} \subset \mathfrak{g}$ (see footnote 14); the embedding $x \mapsto J_{x}: M \rightarrow \mathfrak{g}$ (standard embedding) is extrinsic symmetric (cf. [8], [6]).

Extrinsic symmetric spaces are the most beautiful submanifolds, playing a similar rôle for submanifold geometry as symmetric spaces do for Riemannian geometry. While the latter spaces have parallel curvature tensor, the former ones have parallel second fundamental form which follows from the invariance of $\nabla \alpha$ under $\tau_{x *} \cdot{ }^{8}$ The Gauss equations (1) show that $\alpha$ is even more fundamental than $R$. As symmetric spaces are stable in the sense of "intrinsic pinching" (a Riemannian manifold with $\nabla R \approx 0$ is already diffeomorphic

\footnotetext{
${ }^{7}$ Cf. [3] for details and further examples.

${ }^{8} \nabla \alpha(u, v, w)=\tau_{x *} \nabla \alpha(u, v, w)=\nabla \alpha\left(\tau_{x *} u, \tau_{x *} v, \tau_{x *} w\right)=-\nabla \alpha(u, v, w)$.
} 
to a locally symmetric space, [13], [10]), extrinsic symmetric submanifolds are stable in the sense of "extrinsic pinching" [16]: any submanifold with $\nabla \alpha \approx 0$ is already close to an extrinsic symmetric one.

Now let us consider the Gauss map of an extrinsic symmetric space $M \subset$ $\mathrm{E}$. We may assume that $M$ is contained in the unit sphere ${ }^{9} \mathrm{~S} \subset \mathrm{E}$. Then we have $x \in N_{x}$ for all $x \in M$ and thus $\tau_{x}$ is linear, $\tau_{x}=\tau_{x *}$. Since $M$ is invariant under $\tau_{x}$, the same is true for the set of all normal spaces of $M$. But $\mathrm{Gr}$ is a symmetric space whose symmetry at $N_{x} \in \mathrm{Gr}$ is precisely the reflection $\tau_{x}$, therefore the Gauss image $Q=N(M)$ is a symmetric subspace ${ }^{10}$ of Gr. Since the kernel of $d N=-A$ would be a parallel distribution on $M$ whose leaves are affine subspaces of $\mathrm{E}$, the kernel must be zero, thus $N: M \rightarrow Q$ is a covering map (in many cases a diffeomorphism). Moreover it is equivariant with respect to the group

$$
K=\{A \in O(n) ; A(M)=M\}
$$

acting transitively on $M$, and therefore $N$ is an isometry (up to scaling) on each isotropy irreducible component ${ }^{11}$ of $M$. In the case of the sphere $M=\mathrm{S}^{m}$, the Gauss map $N$ identifies $\mathrm{S}^{m}$ with the Grassmannian of oriented one-dimensional subspaces in $\mathrm{E}^{m+1}$.

\section{$5 \quad$ Algebra of extrinsic symmetric spaces}

According to work of Ferus (cf. [8] or [4]), each irreducible extrinsic symmetric space (up to congruence and rescaling) is a certain orbit of the isotropy

\footnotetext{
${ }^{9}$ Since $\alpha$ is parallel, the same holds for the mean curvature vector $\eta=$ trace $\alpha$ and for the corresponding Weingarten map $A_{\eta}$. Hence the eigenspaces of $A_{\eta}$ are also parallel and moreover invariant under any $A_{\xi}$, due to the parallelity of $\eta$ and the Ricci equation which implies $\left[A_{\xi}, A_{\eta}\right]=0$. The leaves of each eigenspace distribution are submanifolds in a subspace of $\mathrm{E}$ and $M$ decomposes into such submanifolds which are the intersections of $M$ with an orthogonal decomposition of $\mathrm{E}$ (extrinsic splitting). Each factor can be considered seperately. If it is not an affine subspace, we have $A_{\eta}=\lambda I$ for some nonzero $\lambda$, and thus $M$ lies in some sphere of radius $1 /|\lambda|$. Normalizing we may assume that $M$ is contained in the unit sphere $\mathrm{S}^{n-1}$.

${ }^{10}$ If $P$ is a symmetric space with symmetries $\sigma_{p}, p \in P$, and if $Q \subset M$ is a connected subset which is invariant under each $\sigma_{q}, q \in Q$, then $Q$ is a symmetric subspace, i.e. a complete totally geodesic submanifold of $P$, see [9] or [3].

${ }^{11}$ In fact, if $M$ does not split extrinsically as $M_{1} \times M_{2} \subset \mathrm{E}^{n_{1}} \times \mathrm{E}^{n_{2}}=\mathrm{E}$ with $M_{i} \subset$ $\mathrm{E}^{n_{i}}$ (extrinsic irreducibilty), it is also intrinsically isotropy irreducible, i.e. the isotropy representation is irreducible ([4], Theorem 4).
} 
representation of another symmetric space as follows. ${ }^{12}$ Let $G$ be a compact Lie group with two commuting involutions $\sigma$ and $\tau$. Then we have a common decomposition of the Lie algebra

$$
\mathfrak{g}=\mathfrak{k}_{-}+\mathfrak{k}_{+}+\mathfrak{p}_{-}+\mathfrak{p}_{+}
$$

where $\mathfrak{k}=\mathfrak{k}_{+}+\mathfrak{k}_{-}$and $\mathfrak{g}_{+}=\mathfrak{k}_{+}+\mathfrak{p}_{+}$are the Lie subalgebras fixed by $\sigma_{*}$ and $\tau_{*}$, respectively. Further we require that $\tau$ is inner and of a very simple type: $\tau_{*}=e^{\pi \operatorname{ad}(\eta)}$ for some $\eta \in \mathfrak{p}_{+}$with $\operatorname{ad}(\eta)^{3}=-\operatorname{ad}(\eta)$, i.e. the eigenvalues of $\operatorname{ad}(\eta)$ are 0 and $\pm i$. More precisely, since $\tau_{*}=e^{\pi \operatorname{ad}(\eta)}=\left\{\begin{array}{ccc}I & \text { on } & \mathfrak{g}_{+} \\ -I & \text { on } & \mathfrak{g}_{-}\end{array}\right.$,

$$
\operatorname{ad}(\eta)=\left\{\begin{array}{lll}
0 & \text { on } & \mathfrak{g}_{+} \\
J & \text { on } & \mathfrak{g}_{-}
\end{array}\right.
$$

where $J$ is a complex structure on $\mathfrak{g}_{+}$(eigenvalues $\pm i$ ). Let $K \subset G$ be the fixed group of $\sigma$. Then $M=\operatorname{Ad}(K) \eta \subset \mathfrak{p}=$ : $\mathrm{E}$ is an extrinsic symmetric space whose normal reflection at the point $\eta$ is $\tau_{*}$. The tangent and normal spaces of $M$ at $\eta$ are $\mathfrak{p}_{-}$and $\mathfrak{p}_{+}$. The ambient space $\mathrm{E}=\mathfrak{p}$ is a Lie triple, i.e. $[\mathfrak{p},[\mathfrak{p}, \mathfrak{p}]] \subset \mathfrak{p}$. The corresponding trilinear map on $\mathfrak{p}$,

$$
R(u, v) w=[w,[u, v]]
$$

is called Lie triple product; it is the curvature tensor of the symmetric space $G / K$ whose tangent space at $e K$ can be identified with $\mathfrak{p}$. Both subspaces $\mathfrak{p}_{+}, \mathfrak{p}_{-}$(being the fixed sets of the involutions $\tau_{*}$ and $\sigma_{*} \tau_{*}$ on $\mathfrak{p}$ ) are Lie subtriples, i.e. they are preserved by $R$. The Lie algebra $\mathfrak{k}$ consists of the derivations ${ }^{13}$ of $R$, and $\mathfrak{k}_{+}$(resp. $\mathfrak{k}_{-}$) contains those elements of $\mathfrak{k}$ which preserve (resp. reverse) the splitting $\mathfrak{p}=\mathfrak{p}_{-}+\mathfrak{p}_{+}$.

The same $\eta \in \mathfrak{p}_{+} \subset \mathfrak{g}_{+}$(cf. (4)) generates yet another extrinsic symmetric space: the full adjoint orbit $\hat{M}=A d(G) \eta \subset \mathfrak{g}$ with $T_{\eta} \hat{M}=\mathfrak{g}_{-}$and $N_{\eta} \hat{M}=\mathfrak{g}_{+}$. The complex structure $J$ on $\mathfrak{g}_{-}$defined in (4) makes $\hat{M}$ hermitian symmetric, and $\hat{M} \subset \mathfrak{g}$ is the standard embedding mentioned above. Thus we see that any extrinsic symmetric space $M$ lies in a standard embedded hermitian symmetric space $\hat{M}$; in fact, $M$ is a real form of $\hat{M}$ since it is the fixed set of $\left.\sigma_{*}\right|_{\hat{M}}$ which acts as a complex conjugation on $\hat{M}$.

\footnotetext{
${ }^{12}$ Recently, J.R. Kim gave a similar characterization for extrinsic symmetric spaces with indefinite inner product, cf. [11], [5].

${ }^{13}$ Derivations of $R$ are skew adjoint linear maps $A: \mathfrak{p} \rightarrow \mathfrak{p}$ with $A R(x, y) z=R(A x, y) z+$ $R(x, A y) z+R(x, y) A z$. They form the Lie algebra of the automorphism group $K$ of $\mathfrak{p}$.
} 
We can also view the hermitian case as a special case of the general construction of extrinsic symmetric spaces as follows. To avoid confusion with the notation in the general case we change symbols renaming $\mathfrak{g} \rightarrow \mathfrak{h}$, $J \rightarrow j, \eta \rightarrow \zeta$. The compact Lie group $H$ (previous $G$ ) is considered as a symmetric space $H=G / K$ with $G=H \times H$ and $K=\{(h, h) ; h \in H\}$. Therefore we put $\mathfrak{g}=\mathfrak{h} \oplus \mathfrak{h}$ and

$$
\mathfrak{p}=\{(X,-X) ; X \in \mathfrak{h}\}, \quad \mathfrak{k}=\{(X, X) ; X \in \mathfrak{h}\} .
$$

The Cartan decomposition $\mathfrak{h}=\mathfrak{h}_{+}+\mathfrak{h}_{-}$extends to $\mathfrak{g}=\mathfrak{g}_{+}+\mathfrak{g}_{-}$, and we have $\eta=(\zeta,-\zeta) \in \mathfrak{p}_{+}$and $J=(j,-j)$.

\section{The second fundamental form}

Now let $M_{o} \subset \mathrm{E}=\mathfrak{p}$ be an irreducible extrinsic symmetric space and $M \subset \mathrm{E}$ any submanifold of the same dimension with Gauss image $N(M) \subset Q:=$ $N\left(M_{o}\right)$. We fix some point $x \in M$ and let $\eta \in M_{o}$ such that $N_{x} M=$ $N_{\eta} M_{o}=\mathfrak{p}_{+}$and $T_{x} M=T_{\eta} M_{o}=\mathfrak{p}_{-}$. For tangent and normal vector fields $v, w$ and $\xi$ on $M$ we consider the Levi-Civita derivatives

$$
\nabla_{v} w=\left(\partial_{v} w\right)^{T}, \quad \nabla_{v} \xi=\left(\partial_{v} \xi\right)^{N}
$$

Together they form a covariant derivative on the trivial bundle $M \times \mathrm{E}=$ $T M \oplus N M$. We show first that the constant Lie triple product $R$ is parallel also with respect to this derivative:

Lemma $1 \nabla R=0$.

Proof. Let $a, b, c, d$ be $\nabla$-parallel vector fields along any curve $x(t)$ in $M$; we may assume that each of them is either tangent or normal. Put $R(a, b, c, d):=$ $\langle R(a, b) c, d\rangle$. Denoting ' $=\frac{d}{d t}$ we have to show $R(a, b, c, d)^{\prime}=0$. But

$$
R(a, b, c, d)^{\prime}=R\left(a^{\prime}, b, c, d\right)+R\left(a, b^{\prime}, c, d\right)+R\left(a, b, c^{\prime}, c\right)+R\left(a, b, c, d^{\prime}\right) .
$$

Since both the tangent and normal space, $\mathfrak{p}_{-}$and $\mathfrak{p}_{+}$, are Lie subtriples, we have $R(a, b, c, d)=0$ unless $R$ has an even number of entries of each type (tangent vs. normal); e.g. $R\left(\mathfrak{p}_{-}, \mathfrak{p}_{-}, \mathfrak{p}_{-}, \mathfrak{p}_{+}\right)=0$ since $R\left(\mathfrak{p}_{-}, \mathfrak{p}_{-}\right) \mathfrak{p}_{-} \subset \mathfrak{p}_{-} \perp$ $\mathfrak{p}_{+}$, and by the curvature identities this remains true with permuted entries. 
But the derivative of a $\nabla$-parallel tangent (resp. normal) field is normal (resp. tangent), hence each term on the right hand side contains an odd number of entries of each sort, thus it has to be zero.

The difference tensor between the two derivatives $\nabla$ and $\partial$ on $M \times E$,

$$
L:=\partial-\nabla
$$

is essentially the second fundamental form; we have

$$
L_{v} w=\alpha(v, w), \quad L_{v} \xi=-A_{\xi} v .
$$

Since $\partial_{v} R=0$ and $\nabla_{v} R=0$ by the previous lemma, we get $L_{v} R=0$, i.e. $L_{v}$ is a derivation of $R$ and therefore ${ }^{14} L_{v} \in \mathfrak{k}$, acting as ad $\left(L_{v}\right)$. More precisely, $L_{v}$ interchanges $\mathfrak{p}_{-}$and $\mathfrak{p}_{+}$, hence $L_{v} \in \mathfrak{k}_{-}$. Thus we have defined a linear map

$$
L: \mathfrak{p}_{-} \rightarrow \mathfrak{k}_{-}, \quad v \mapsto L_{v}=L v .
$$

Let $L^{t}: \mathfrak{k}_{-} \rightarrow \mathfrak{p}_{-}$be the transposed of $L$ with respect to an $\operatorname{Ad}(G)$-invariant inner product on $\mathfrak{g}$ extending the given $\operatorname{Ad}(K)$-invariant inner product on $\mathfrak{p}=\mathrm{E}$.

Lemma 2 For all $\xi \in \mathfrak{p}_{+}$we have

$$
\begin{aligned}
L^{t} \circ \operatorname{ad}(\xi) & =-\operatorname{ad}(\xi) \circ L, \\
L \circ \operatorname{ad}(\xi) & =-\operatorname{ad}(\xi) \circ L^{t} .
\end{aligned}
$$

Proof. The first equation follows from the symmetry of $\alpha$ which reads

$$
[L v, w]=[L w, v]
$$

for all $v, w \in \mathfrak{p}_{-}$. In fact, for any $\xi \in \mathfrak{p}_{+}$we have

$$
\begin{aligned}
& \langle[L v, w], \xi\rangle=\langle L v,[w, \xi]\rangle=-\left\langle v, L^{t} \operatorname{ad}(\xi) w\right\rangle \\
& \langle[L w, v], \xi\rangle=\langle v,[\xi, L w]\rangle=\langle v, \operatorname{ad}(\xi) L w\rangle
\end{aligned}
$$

\footnotetext{
${ }^{14}$ Any Lie triple derivation $L: \mathfrak{p} \rightarrow \mathfrak{p}$ extends uniquely to a Lie algebra derivation $L: \mathfrak{g} \rightarrow \mathfrak{g}$ where for every $A \in \mathfrak{k}$ we let $L(A) \in \mathfrak{k}$ with $[L(A), x]=L[A, x]-[A, L x]$ for any $x \in \mathfrak{p}$. Since $\mathfrak{g}$ is semisimple, each derivation is inner, $L=\operatorname{ad}(l)$ for some $l \in \mathfrak{g}$. In fact $l \in \mathfrak{k}$ since $L=\operatorname{ad}(l)$ preserves $\mathfrak{p}$ and $\mathfrak{k}$.
} 
which proves (11). The second equation (12) is obtained by composing (11) from both sides with $\operatorname{ad}(\eta)$. From (4) we see that $\operatorname{ad}(\eta)$ and $\operatorname{ad}(\xi)$ commute (since $[\operatorname{ad}(\eta), \operatorname{ad}(\xi)]=\operatorname{ad}([\eta, \xi])=0)$, and further $\operatorname{ad}(\eta)^{2}=J^{2}=-I$ on $\mathfrak{g}_{-}$. Using (11) for $\eta$ in place of $\xi$,

$$
\begin{aligned}
\operatorname{ad}(\eta) L^{t} \operatorname{ad}(\xi) \operatorname{ad}(\eta) & =\operatorname{ad}(\eta) L^{t} \operatorname{ad}(\eta) \operatorname{ad}(\xi)=-\operatorname{ad}(\eta)^{2} L \operatorname{ad}(\xi)=L \operatorname{ad}(\xi), \\
-\operatorname{ad}(\eta) \operatorname{ad}(\xi) L \operatorname{ad}(\eta) & =-\operatorname{ad}(\xi) \operatorname{ad}(\eta) L \operatorname{ad}(\eta)=\operatorname{ad}(\xi) L^{t} \operatorname{ad}(\eta)^{2}=-\operatorname{ad}(\xi) L^{t}
\end{aligned}
$$

The left hand sides are equal by (11), hence we have proved (12).

The second fundamental form $L$ defines a skew symmetric linear map $\hat{L}: \mathfrak{g}_{-} \rightarrow \mathfrak{g}_{-}$interchanging $\mathfrak{p}_{-}$and $\mathfrak{k}_{-}$:

$$
\hat{L}=\left\{\begin{array}{ccc}
L & \text { on } & \mathfrak{p}_{-} \\
-L^{t} & \text { on } & \mathfrak{k}_{-}
\end{array}\right.
$$

Lemma $3 \hat{L}$ commutes with the action of $\tilde{\mathfrak{g}}_{+}=\tilde{\mathfrak{k}}_{+}+\mathfrak{p}_{+}$on $\mathfrak{g}_{-}$, where $\tilde{\mathfrak{k}}_{+}=$ $\left[\mathfrak{p}_{+}, \mathfrak{p}_{+}\right] \subset \mathfrak{k}_{+}$.

Proof. From Equations (11) and (12) of the previous Lemma we obtain

$$
\hat{L} \circ \operatorname{ad}(\xi)=\operatorname{ad}(\xi) \circ \hat{L}
$$

for all $\xi \in \mathfrak{p}_{+}$. Hence $\hat{L}$ commutes with the action of $\mathfrak{p}_{+}$on $\mathfrak{g}_{-}$, and consequently with the action of $\left[\mathfrak{p}_{+}, \mathfrak{p}_{+}\right]=\tilde{\mathfrak{k}}_{+}$.

\section{The group action}

Lemma 4 Let $M_{o} \subset \mathfrak{p}$ be extrinsic symmetric and irreducible, but $M_{o} \neq \mathrm{S}^{m}$. Then $\left[\mathfrak{p}_{+}, \mathfrak{p}_{+}\right]=\mathfrak{k}_{+}$and hence $\tilde{\mathfrak{g}}_{+}=\mathfrak{g}_{+}$.

Proof. Note that any $A \in \mathfrak{k}_{+}$with $A \perp\left[\mathfrak{p}_{+}, \mathfrak{p}_{+}\right]$acts trivially on $\mathfrak{p}_{+}$. Hence $\left(\tilde{\mathfrak{g}}_{+}, \tilde{\mathfrak{k}}_{+}\right)$is the effective pair ${ }^{15}$ corresponding to the Lie triple $\mathfrak{p}_{+}$. We show by inspection of the tables for $M_{o}$ (cf. [14], p. $241 \mathrm{f}$ ) that

$$
\mathfrak{k}_{+}=\tilde{\mathfrak{k}}_{+}=\left[\mathfrak{p}_{+}, \mathfrak{p}_{+}\right]
$$

\footnotetext{
${ }^{15}$ We call the pair of Lie algebras $(\mathfrak{g}, \mathfrak{k})$ with $\mathfrak{k} \subset \mathfrak{g}$ ineffective if there is a nonzero subalgebra $\mathfrak{l} \subset \mathfrak{k}$ with $[\mathfrak{l}, \mathfrak{g}] \subset \mathfrak{k}$; in other words, the subgroup $L \subset K$ corresponding to $\mathfrak{l}$ acts trivially on $G / K$.
} 
in all cases but the one corresponding to $M_{o}=\mathrm{S}^{m}$ (Case No. 13 for $i=1$ ). In fact, the symmetric pairs $\left(\mathfrak{k}, \mathfrak{k}_{+}\right)$corresponding to $\mathfrak{k}_{-}$are listed on p. 242, first column while the last column contains the pairs $\left(\tilde{\mathfrak{g}}_{+}, \tilde{\mathfrak{k}}_{+}\right)$corresponding to $\mathfrak{p}_{+} \cdot{ }^{16}$ We have $\tilde{\mathfrak{k}}_{+}=\mathfrak{k}_{+}$in all cases except No. 13 for $i=1$ where $\mathfrak{p}_{+}=\mathbb{R}$ and the pair $(\mathbb{R}+\mathfrak{s o}(n-2), \mathfrak{s o}(n-2))$ is ineffective; the effective one would be $\left(\tilde{\mathfrak{g}}_{+}, \tilde{\mathfrak{k}}_{+}\right)=(\mathbb{R}, 0)$.

\section{Lemma 5}

$$
\hat{L}=\lambda \operatorname{ad}(\eta)
$$

for some real $\lambda$.

Proof. The vector space $\mathfrak{g}_{-}$has a complex structure $J=\left.\operatorname{ad}(\eta)\right|_{\mathfrak{g}_{-}}$which commutes with the action of $\mathfrak{g}_{+}$(by (4)) and of $\hat{L}$ (by (15)), i.e. these actions are complex linear.

If $M_{o}$ belongs to the cases 7-18 in [14] or [2], the Lie algebra $\mathfrak{g}$ is simple and hence the symmetric pair $\left(\mathfrak{g}, \mathfrak{g}_{+}\right)$is irreducible. ${ }^{17}$ Thus $\mathfrak{g}_{+}$acts irreducibly on $\mathfrak{g}_{-}$, and $\hat{L}$ commutes with this action. By Schur's lemma, $\hat{L}=\alpha I$ for some $\alpha \in \mathbb{C}$. But $\hat{L}$ is antisymmetric as a real endomorphism, hence $\alpha$ is purely imaginary, $\alpha=\lambda i$ and $\hat{L}=\lambda J=\left.\lambda \operatorname{ad}(\eta)\right|_{\mathfrak{g}_{-}}$.

The cases $1-6$ are those where $M_{o}$ is hermitian and $\mathfrak{p}$ itself a Lie algebra. More precisely, there is a simple compact Lie algebra $\mathfrak{h}$ with Cartan decomposition $\mathfrak{h}=\mathfrak{h}_{+}+\mathfrak{h}_{-}$such that $\mathfrak{g}=\mathfrak{h} \oplus \mathfrak{h}$ and

$$
\mathfrak{p}=\{(X,-X) ; X \in \mathfrak{h}\}, \quad \mathfrak{k}=\{(X, X) ; X \in \mathfrak{h}\} .
$$

Now $\mathfrak{g}_{-}=\mathfrak{h}_{-} \oplus \mathfrak{h}_{-}$has two irreducible factors for the action of $\mathfrak{g}_{+}=\mathfrak{h}_{+} \oplus \mathfrak{h}_{+}$, and the complex structure $J=(j,-j)$ is given by the Lie bracket with $\eta=(\zeta,-\zeta) \in \mathfrak{p}_{+}$. By Schur's lemma, $\hat{L}$ has (imaginary) eigenvalues $\lambda i, \mu i$ on the two irreducible factors, and in particular

$$
\hat{L}(X, X)=(\lambda j X,-\mu j X)
$$

for all $X \in \mathfrak{h}_{-}$. But on the other hand $\hat{L}\left(\mathfrak{k}_{-}\right) \subset \mathfrak{p}_{-}$, hence

$$
\hat{L}(X, X)=(Y,-Y)
$$

for some $Y \in \mathfrak{h}_{-}$. Comparing these equations shows $\lambda=\mu$.

\footnotetext{
${ }^{16}$ The table in [2], p. $735 \mathrm{f}$ is very similar: It displays the pairs $\left(\mathfrak{k}, \mathfrak{k}_{+}\right)$on p. 735 , third column and the noncompact duals of $\left(\tilde{\mathfrak{g}}_{+}, \tilde{\mathfrak{k}}_{+}\right)$on p. 736 , second column

${ }^{17}$ see [14], p. 241, third column or the dual in [2], p. 736, first column
} 


\section{Lemma 6}

$$
\lambda=\text { const }
$$

Proof. Locally we may view $\lambda$ and $\eta$ as smooth functions on $M$. Since $\eta$ is the position vector of $M_{o} \subset \mathrm{S}$, its derivatives $\partial_{v} \eta$ lie in $T_{\eta} M_{o}=T_{x} M$, hence $\eta$ is a parallel normal vector field on $M$. By Lemma 1 we have $\nabla R=0$, and hence $\operatorname{ad}(\eta)=R(., \eta): \mathfrak{p}_{-} \rightarrow \mathfrak{k}_{-} \subset \operatorname{Hom}\left(\mathfrak{p}_{+}, \mathfrak{p}_{-}\right)$is $\nabla$-parallel. ${ }^{18}$ Codazzi equations show

$$
\left(\nabla_{v} L\right) w=\left(\nabla_{w} L\right) v
$$

for any two tangent vectors $v, w \in \mathfrak{p}_{-}$. Using (17) and (4) we obtain

$$
\left(\partial_{v} \lambda\right) J w=\left(\partial_{w} \lambda\right) J v .
$$

If $v, w$ are linearly independent we have $\partial_{v} \lambda=0=\partial_{w} \lambda$. Thus $\lambda=$ const.

Proof of the Theorem. If $\lambda=0$, then $L=0$ and our submanifold $M$ is affine. Otherwise we may assume $\lambda= \pm 1$ (up to scaling), and replacing $\eta$ by $-\eta$ if necessary, we have $\lambda=1$. Since $\operatorname{ad}(\eta): \mathfrak{p}_{-} \rightarrow \mathfrak{k}_{-} \subset \operatorname{Hom}\left(\mathfrak{p}_{+}, \mathfrak{p}_{-}\right)$ is a linear isometry up to scaling, being the differential of the (equivariant) Gauss map of $M_{o}$, the same holds for $L$. Thus both Gauss maps $N: M \rightarrow Q$ and $N_{o}: M_{o} \rightarrow Q$ are local isometries, and using these maps to identify the abstract Riemannian manifold $M$ with an open subset of $M_{o}$, the second fundamental forms are the same $(\hat{L}=\operatorname{ad}(\eta))$. Now the theorem follows from the uniqueness part of the existence and uniqueness theorem for submanifolds.

\section{References}

[1] J. Berndt, S. Console, C. Olmos: Submanifolds and Holonomy, CRC Press 2003

[2] J. Berndt, J.-H. Eschenburg, H. Naitoh, K. Tsukada: Symmetric submanifolds associated with irreducible symmetric R-spaces, Math. Ann. 332 (2005), 721-737

[3] J.-H. Eschenburg: Lecture Notes on Symmetric Spaces, Preprint Augsburg 2000

\footnotetext{
${ }^{18}$ Remind that $\mathfrak{p}_{-}, \mathfrak{p}_{+}$now depend on $x \in M$; they are subbundles of $M \times \mathrm{E}$.
} 
[4] J.-H. Eschenburg, E. Heintze: Extrinsic symmetric spaces and orbits of s-representations, manuscripta math. 88 (1995), 517-524, Erratum manuscripta math. 92 (1997), 408

[5] J.-H. Eschenburg, J.R. Kim: Indefinite Extrinsic Symmetric Spaces, in preparation

[6] J.-H. Eschenburg, P. Quast: Pluriharmonic maps into Kähler symmetric spaces, Preprint Augsburg 2006

[7] J.-H. Eschenburg, R. Tribuzy: Associated families of pluriharmonic maps and isotropy, manuscripta math. 95 (1998), 295-310

[8] D.Ferus: Symmetric submanifolds of Euclidean space, Math. Ann. 246 (1980), 81-93

[9] S. Helgason: Differential Geometry, Lie Groups and Symmetric Spaces, Academic Press, 1978

[10] A. Katsuda, A pinching problem for locally homogeneous spaces. J. Math. Soc. Japan 41 (1989) 57-74

[11] J.R. Kim: Indefinite Extrinsic Symmetric Spaces, Thesis Augsburg 2005

[12] S. Kobayashi, T. Nagano: On filtered Lie algebras and geometric structures, I, J. Math. Mech. 13 (1964) 875-907.

[13] M. Min-Oo, E.A. Ruh: Comparison theorems for compact symmetric spaces. Ann. sc. Éc. Norm. Sup. (4)12 (1979), 335-353

[14] H. Naitoh: Symmetric submanifolds of compact symmetric spaces, Tsukuba J. Math. 10 (1986), 215-224

[15] Y.A. Nikolaevskii: Classification of Multidimensional Submanifolds in Euclidian Space with Totally Geodesic Gauss Image, Russian Academy of Sciences Sbornik Mathematics, 76 (1993), 225-246

[16] Quast, Peter: A pinching theorem for extrinsically symmetric submanifolds of Euclidean space. Manuscr. Math. 115 (2004), 427-436

[17] E. Ruh, J. Vilms: The tension field of the Gauss map, Trans. Amer. Math. Soc. 149 (1970), 569 - 573 